\title{
Comment on "Smoking prevalence and effects on treatment outcomes in patients with tuberculosis"
}

\author{
Liwen Liu ${ }^{1}$, Xiaofei Li *
}

Dear Editor,

We were very pleased to read the article "Smoking prevalence and effects on treatment outcomes in patients with tuberculosis" by Vargas and colleagues ${ }^{1}$. In this study, the authors revealed that active smokers had less chance for cure and more abandonment than non-active smokers. This study provides very valuable insight for tuberculosis (TB) control, especially in cognitive-behavioral approaches to smoking cessation. However, some concerns should be raised from our point of view.

First, though smoking status was determined according to the definitions of the Centers for Disease Control and Prevention, a brief description of smoking status should also be provided in the Materials and methods section. There is an apparent sex difference among active smoking patients involved in this study. Thus, sex-stratified analysis could reduce the impact of sex on the results. These results were influenced not only by the subjects' gender but also by smoking history.

Second, the reliability and validity of the Fagerström scale have not been assessed in this study. Also, the statistical values should be provided. Further studies are needed to determine more sociodemographic characteristics, including age, occupation, education level, ethnicity, parity, and residency. It may be found that more vital risk factors also play an important role in the control of TB.

\section{AUTHORS' CONTRIBUTIONS}

LL: Data curation, Formal Analysis, Writing - original draft. XL: Conceptualization, Writing - review and editing.

\section{REFERENCE}

1. Vargas KR, Freitas AA, Azeredo ACV, Silva DR. Smoking prevalence and effects on treatment outcomes in patients with tuberculosis. Rev Assoc Med Bras (1992). 2021;67(3):406-10. https://doi.org/10.1590/1806-9282.20200825

${ }^{1}$ Yiwu Central Hospital, Department of Infectious Diseases - Zhejiang, China.

*Corresponding author: xiaofeili2000@163.com

Conflicts of interest: the authors declare there are no conflicts of interest. Funding: none.

Received on August 15, 2021. Accepted on August 15, 2021. 